\title{
Minimal geometric deformation in asymptotically (A-)dS space-times and the isotropic sector for a polytropic black hole
}

\author{
Ernesto Contreras ${ }^{1, \mathrm{a}}$, Pedro Bargueño ${ }^{2, \mathrm{~b}}$ \\ ${ }^{1}$ School of Physical Sciences and Nanotechnology, Yachay Tech University, Urcuquí 100119, Ecuador \\ 2 Departamento de Física, Universidad de los Andes, Apartado Aéreo 4976, Bogotá, Distrito Capital, Colombia
}

Received: 2 October 2018 / Accepted: 20 November 2018 / Published online: 30 November 2018

(C) The Author(s) 2018

\begin{abstract}
In the context of the minimal geometric deformation method, in this paper we implement the inverse problem in a black hole scenario. In order to deal with an anisotropic polytropic black hole solution of the Einstein field equations with cosmological constant, the deformation method is slightly extended. After obtaining the isotropic sector and the decoupler for an anisotropic (A-)dS polytropic black hole solution, we emphasize a possible relation between anisotropization/isotropization and the violation of the energy conditions.
\end{abstract}

\section{Introduction}

In recent years, the use of the minimal geometric deformation (MGD) [1-33] as a systematic and powerful method to obtain new and relevant solutions of the Einstein field equations, has considerable increased [18,22-27,30,32]. For example, the method has allowed to induce local anisotropies in spherically symmetric systems leading to both more realistic interior solutions of compact objects $[25,26]$ and hairy black holes [24]. More recently, the method has been extended to solve the inverse problem [32], namely, given any anisotropic solution of the Einstein field equations it is possible to recover the isotropic source and the decoupler matter content which, after gravitational interaction, led to the anisotropic configuration. In that work, it was found that, for a simple anisotropic solution violating all the energy conditions, the free parameters involved in the MGD can be fitted in such a manner that both the isotropic source and the matter decoupler content satisfy all the energy conditions. The importance of this result lies in the fact that the inverse problem allowed to inter-

E. Contreras: On leave from Universidad Central de Venezuela.

a e-mail: econtreras@yachaytech.edu.ec; ernesto.contreras@ciens.ucv.ve

b e-mail: p.bargueno@uniandes.edu.co pret the MGD as some kind of mechanism which leads to the apparition of exotic matter after gravitational interaction of well behaved matter content.

In the same spirit of Ref. [32], it could be interesting to explore if the same duality exotic/non-exotic matter content occurs in other scenarios after the application of the inverse MGD problem. To be more precise, we could study if such a duality persists in situations where the starting point is a solution sourced by a matter content satisfying all the energy conditions. In order to do so, in this work we implement the inverse problem program in a polytropic black hole (BH) originally studied in reference [34] and extended to the scaledependent scenario in Ref. [35]. As it will be shown in the rest of the manuscript, the choice of such a system is twofold: first, to extend the MGD in order to deal with Einstein field equations with cosmological constant and, second, to implement the inverse problem in an anisotropic system which satisfies all the energy conditions.

This work is organized as follows. In the next section we briefly review the MGD-decoupling method. In Sect. 3 we develop the method to obtain the generator of any anisotropic solution of the Einstein Field Equations and then we implement the method for a polytropic BH solution in Sect. 4. The last section is devoted to final comments and conclusion.

\section{Einstein equations with cosmological constant and extended MGD-decoupling}

With the purpose to extend the MGD in order to consider the Einstein field equations with cosmological constant, we write

$R_{\mu \nu}-\frac{1}{2} R g_{\mu \nu}+\Lambda g_{\mu \nu}=-\kappa^{2} T_{\mu \nu}^{t o t}$,

and we assume that the total energy-momentum tensor is given by 
$T_{\mu \nu}^{(t o t)}=T_{\mu \nu}^{(m)}+\alpha \theta_{\mu \nu}$,

where $\alpha$ is a constant. As usual, the energy-momentum tensor for a perfect fluid $T_{\nu}^{\mu(m)}=\operatorname{diag}(-\rho, p, p, p)$ and the decoupler matter content $\theta_{\mu \nu}$ interact only gravitationally,

$\nabla_{\mu} T_{\nu}^{\mu(m)}=\nabla_{\mu} \theta_{\nu}^{\mu}=0$.

In what follows, we shall work with spherically symmetric space-times with a line element parametrized as

$d s^{2}=e^{v} d t^{2}-e^{\lambda} d r^{2}-r^{2} d \Omega^{2}$

where $v$ and $\lambda$ are functions of the radial coordinate $r$ only. Considering Eq. (4) as a solution of the Einstein field equations, we obtain

$$
\begin{aligned}
\kappa^{2} \tilde{\rho} & =\frac{e^{-\lambda}\left(r \lambda^{\prime}-1\right)}{r^{2}}+\frac{\Lambda r^{2}+1}{r^{2}} \\
\kappa^{2} \tilde{p}_{r} & =\frac{e^{-\lambda}\left(r v^{\prime}+1\right)}{r^{2}}-\frac{1}{r^{2}}-\Lambda \\
\kappa^{2} \tilde{p}_{\perp} & =-\Lambda-\frac{e^{-\lambda}\left(\left(r v^{\prime}+2\right)\left(\lambda^{\prime}-v^{\prime}\right)-2 r v^{\prime \prime}\right)}{4 r}
\end{aligned}
$$

where the prime denotes derivation with respect to the radial coordinate and we have defined

$$
\begin{aligned}
& \tilde{\rho}=\rho+\alpha \theta_{0}^{0} \\
& \tilde{p}_{r}=p-\alpha \theta_{1}^{1} \\
& \tilde{p}_{\perp}=p-\alpha \theta_{2}^{2} .
\end{aligned}
$$

The next step consists in decoupling the Einstein field equations (5), (6) and (7) by performing

$e^{-\lambda}=\mu+\alpha f$,

where $f$ is the geometric deformation undergone by the radial metric component $\mu$, "controlled" by the free parameter $\alpha$. By doing so, we obtain two sets of differential equations: one describing an isotropic system sourced by the conserved energy-momentum tensor of a perfect fluid $T_{\nu}^{\mu(m)}$ an the other set corresponding to quasi-Einstein field equations sourced by $\theta_{\mu \nu}$. After taking into account that the cosmological constant can be interpreted as some kind of isotropic fluid, we include the $\Lambda$-term in the isotropic sector and we obtain

$$
\begin{aligned}
\kappa^{2} \rho= & \frac{\Lambda r^{2}-r \mu^{\prime}-\mu+1}{r^{2}} \\
\kappa^{2} p= & \frac{-\Lambda r^{2}+r \mu(r) v^{\prime}+\mu-1}{r^{2}} \\
\kappa^{2} p= & \frac{2 \mu^{\prime}+2 r \mu v^{\prime \prime}+r \mu v^{\prime 2}+2 \mu v^{\prime}}{4 r} \\
& +\frac{\mu^{\prime} v^{\prime}}{4}+\Lambda,
\end{aligned}
$$

for the perfect fluid and $\kappa^{2} \theta_{0}^{0}=-\frac{r f^{\prime}+f}{r^{2}}$

$\kappa^{2} \theta_{1}^{1}=-\frac{r f v^{\prime}+f}{r^{2}}$

$\kappa^{2} \theta_{2}^{2}=-\frac{f^{\prime}\left(r v^{\prime}+2\right)+f\left(2 r v^{\prime \prime}+r v^{\prime 2}+2 v^{\prime}\right)}{4 r}$,

for the anisotropic system. ${ }^{1}$ We would like to emphasize that that the addition of the cosmological constant only affects the isotropic sector because Eqs. (15), (16) and (17) remain unchanged. At this point, we are ready to implement the inverse problem program.

\section{MGD-decoupling: the inverse problem}

In a previous work [32], the inverse MGD problem was solved after realizing that, given any anisotropic solution with metric functions $\{v, \lambda\}$, matter content $\left\{\tilde{\rho}, \tilde{p}_{r}, \tilde{p}_{\perp}\right\}$ and definitions given by Eqs. (8), (9) and (10), the following constraint must be satisfied:

$\tilde{p}_{\perp}-\tilde{p}_{r}=-\alpha\left(\theta_{2}^{2}-\theta_{1}^{1}\right)$.

It should be noted that the above constraint allows us to obtain a differential equation for the decoupling function $f$ in terms of well known quantities of anisotropic solution. To be more precise, with this constraint we do not need any artificial equation of state for the $\theta$ 's components. It is worth mentioning that, in the case of Einstein equations with cosmological constant, the previous constraint leads to the same result obtained in Ref. [32] because there is no contribution of $\Lambda$. More precisely, after subtracting Eqs. (9) and (10), the cosmological constant disappears and the solution remains the same. In this sense, the combination of Eqs. (16) and (17) with the constraint (18) leads to a differential equation for the decoupling function $f$ given by

$f^{\prime}-\mathcal{F}_{1} f=\mathcal{F}_{2}$,

where we have introduced the auxiliary functions $\mathcal{F}_{1}$ and $\mathcal{F}_{2}$ as

$$
\begin{aligned}
\mathcal{F}_{1}= & \frac{4-r\left(2 r v^{\prime \prime}+v^{\prime}\left(r v^{\prime}-2\right)\right)}{r\left(r v^{\prime}+2\right)} \\
\mathcal{F}_{2}= & \frac{e^{-\lambda}\left(r\left(-\lambda^{\prime}\left(r v^{\prime}+2\right)+2 r v^{\prime \prime}+v^{\prime}\left(r v^{\prime}-2\right)\right)\right.}{\alpha r\left(r v^{\prime}+2\right)} \\
& +\frac{\left.4 e^{-\lambda}\left(e^{\lambda}-1\right)\right)}{\alpha r\left(r v^{\prime}+2\right)} .
\end{aligned}
$$

From Eq. (19), it is straightforward to derive that the deformation function, $f$, is given by

$f(r)=e^{\int^{r} \mathcal{F}_{1} d u} \int^{r} \mathcal{F}_{2} e^{-\int^{w} \mathcal{F}_{1} d u} d w$.

\footnotetext{
${ }^{1}$ In what follows we shall assume $\kappa^{2}=8 \pi$.
} 
The next step consists in obtaining the metric function, $\mu$, by replacing Eq. (22) in the geometric deformation relation (11). We obtain

$\mu=e^{-\lambda}-\alpha e^{\int^{r} \mathcal{F}_{1} d u} \int^{r} \mathcal{F}_{2} e^{-\int^{w} \mathcal{F}_{2} d u} d w$.

Now, from Eqs. (12), (13) and (14), the matter content for the isotropic system reads

$\rho=\Lambda+\mathcal{G}_{1}+\alpha \mathcal{G}_{2} e^{\int^{r} \mathcal{F}_{1} d u} \int^{r} \mathcal{F}_{2} e^{-\int^{w} \mathcal{F}_{1} d u} d w$

$p=-\Lambda+\mathcal{G}_{3}-\alpha \mathcal{G}_{4} e^{\int^{r} \mathcal{F}_{1} d u} \int^{r} \mathcal{F}_{2} e^{-\int^{w} \mathcal{F}_{1} d u} d w$.

where we have introduced four additional auxiliary functions as

$$
\begin{aligned}
\mathcal{G}_{1}= & \frac{r e^{-\lambda}\left(\left(e^{\lambda}-3\right) v^{\prime}+2 r v^{\prime \prime}+r v^{\prime 2}\right)}{8 \pi r^{2}\left(r v^{\prime}+2\right)} \\
& +\frac{6 e^{-\lambda}\left(e^{\lambda}-1\right)}{8 \pi r^{2}\left(r v^{\prime}+2\right)} \\
\mathcal{G}_{2}= & \frac{6-r\left(2 r v^{\prime \prime}+v^{\prime}\left(r v^{\prime}-3\right)\right)}{8 \pi r^{2}\left(r v^{\prime}+2\right)} \\
\mathcal{G}_{3}= & \frac{e^{-\lambda}\left(-e^{\lambda}+r v^{\prime}+1\right)}{8 \pi r^{2}} \\
\mathcal{G}_{4}= & \frac{r v^{\prime}+1}{8 \pi r^{2}} .
\end{aligned}
$$

To determine the decoupler matter content we simply replace Eqs. (22) and (23) in (15), (16) and (17) to obtain

$$
\begin{aligned}
& \theta_{0}^{0}=-\frac{\left(r \mathcal{F}_{1}+1\right) e^{\int^{r} \mathcal{F}_{1} d u} \int^{r} \mathcal{F}_{2} e^{-\int^{w} \mathcal{F}_{1} d u} d w}{r^{2}} \\
& +\frac{\mathcal{F}_{2}}{r} \\
& \theta_{1}^{1}=-\frac{\mathcal{H}_{1} e^{\int^{r} \mathcal{F}_{1} d u}\left(\int^{r} \mathcal{F}_{2} e^{-\int^{w} \mathcal{F}_{1} d u} d w\right)}{r^{2}} \\
& \theta_{2}^{2}=-\frac{e^{\int^{r} \mathcal{F}_{1} d u}\left(\int^{r} \mathcal{F}_{2} e^{-\int^{w} \mathcal{F}_{1} d u} d w\right)}{4 r} \\
& +\frac{\mathcal{F}_{2}\left(\mathcal{H}_{1}+1\right)}{4 r},
\end{aligned}
$$

where

$$
\begin{aligned}
& \mathcal{H}_{1}=1+r v^{\prime} \\
& \mathcal{H}_{2}=\left(\left(r v^{\prime}+2\right)\left(\mathcal{F}_{1}+v^{\prime}(r)\right)+2 r v^{\prime \prime}(r)\right) .
\end{aligned}
$$

At this point some comments are in order. First, note that the cosmological constant affects only the isotropic sector, as previously said, whereas the anisotropic one remains unchanged with respect to the reported in Ref. [32]. Second, as reported in Ref. [32], Eqs. (23), (24) and (25) determine the isotropic generator $\{\mu, \rho, p\}$ and Eqs. (30), (31) and (32) determine the decoupler matter content $\left\{\theta_{0}^{0}, \theta_{1}^{1}, \theta_{2}^{2}\right\}$ once any anisotropic solution $\left\{v, \lambda, \tilde{\rho}, \tilde{p}_{r}, \tilde{p}_{\perp}\right\}$ is provided.

In the next section, we shall briefly review the mains aspects of the polytropic BH reported in Ref. [34] and then we shall implement the method to obtain its isotropic generator and decoupler matter content.

\section{Isotropic sector of a polytropic BH solution}

Let us start this section summarizing the main results obtained in Ref. [34]. The line element is parametrized as

$d s^{2}=\left(\frac{r^{2}}{L^{2}}-\frac{2 M}{r}\right) d t^{2}-\left(\frac{r^{2}}{L^{2}}-\frac{2 M}{r}\right)^{-1} d r^{2}-r^{2} d \Omega^{2}$,

with $L^{2}=-3 / \Lambda$. Imposing this metric as a solution of the Einstein field equations with cosmological constant (see Eq. (1)) with $T^{\mu \nu}=\operatorname{diag}\left(-\tilde{\rho}, \tilde{p}_{r}, \tilde{p}_{\perp}, \tilde{p}_{\perp}\right)$ it is obtained that

$\tilde{\rho}=-\tilde{p}_{r}=\frac{1}{8 \pi r^{2}}$,

$\tilde{p}_{\perp}=\tilde{p}_{\perp}=0$.

It is remarkable that $T_{\mu \nu}$ fulfils all the energy conditions

$\tilde{\rho} \geq 0$

$$
\tilde{\rho}+\tilde{p}_{i} \geq 0
$$

$\tilde{\rho}+\sum_{i} \tilde{p}_{i} \geq 0$

$\tilde{\rho}+\tilde{p}_{i} \geq 0$,

$\tilde{\rho} \geq\left|\tilde{p}_{i}\right|$,

which are referred as the weak, strong and dominant energy conditions, respectively. It is worth mentioning that the solution is singular at $r=0$ and has both a Killing and a causal horizon located at $r=2^{1 / 3} M^{1 / 3} L^{2 / 3}$.

In what follows, we shall implement the inverse problem protocol to obtain the isotropic generator and the decoupler matter content associated to this polytropic $\mathrm{BH}$ solution. From Eq. (22), the decoupling function, $f$, reads

$f(r)=e^{v}\left(\frac{\left(2^{4 / 3} L^{2 / 3}\right)\left(2 \sqrt{3} \tan ^{-1} A+\log B\right)}{3 \alpha M^{2 / 3}}+6 c_{1}\right)$

where

$A:=\frac{2 \sqrt[3]{2} r}{\sqrt{3} \sqrt[3]{L^{2} M}}+\frac{1}{\sqrt{3}}$ 
$B:=\frac{\left(\frac{2 M r^{3}}{L^{2}}\right)^{1 / 3}+\left(\frac{2 r^{3}}{L^{2}}\right)^{2 / 3}+M^{2 / 3}}{3\left(\sqrt[3]{M}-\sqrt[3]{2} \sqrt[3]{\frac{r^{3}}{L^{2}}}\right)^{2}}$

$e^{v}=\frac{2 M}{r}-\frac{r^{2}}{L^{2}}$

Replacing Eq. (41) in the decoupling equation, $e^{-\lambda}=$ $\mu+\alpha f$, we obtain an expression for the metric function $\mu$ :

$$
\begin{aligned}
\mu= & -\frac{\left(2 L^{2} M-r^{3}\right)\left(22^{2 / 3} \sqrt{3} \tan ^{-1}(A)+2^{2 / 3} \log (B)\right)}{6 r\left(L^{2} M\right)^{2 / 3}} \\
& -\frac{3\left(3 \alpha c_{1}-2\right)\left(2 L^{2} M-r^{3}\right)}{6 L^{2} r} .
\end{aligned}
$$

Using the above result in Eqs. (24) and (25), the isotropic fluid reads

$$
\begin{aligned}
\rho= & \frac{\frac{12 r^{3}}{L^{2}}-15 M}{8 \pi r^{2}\left(\frac{6 r^{3}}{L^{2}}-3 M\right)}+9 \alpha c_{1} C\left(\frac{M}{L}\right)^{2 / 3} \\
& +C\left(22^{2 / 3} \sqrt{3} \tan ^{-1}(A)+2^{2 / 3} \log (B)\right) \\
p= & \frac{2 \sqrt{3} \tan ^{-1}(A)+\log (B)}{\sqrt[3]{2} 8 \pi\left(L^{2} M\right)^{2 / 3}}+\frac{9 \alpha c_{1} r^{2}}{16 \pi L^{2} r^{2}}-\frac{1}{8 \pi r^{2}}
\end{aligned}
$$

where

$$
C:=\frac{9\left(M-\frac{2 r^{3}}{L^{2}}\right)}{48 \pi(L M)^{2 / 3}\left(\frac{6 r^{3}}{L^{2}}-3 M\right)}
$$

At this point some comments are in order. First, the horizon of the solution is located at

$r_{H}=\sqrt[3]{2} L^{2 / 3} \sqrt[3]{M}$

which coincides with that of the anisotropic solution of Eq. (35). We think this is remarkable: starting from an isotropic solution with an event horizon, one can form, following the MGD, the anisotropic extension of the $\mathrm{BH}$ but maintaining the location of the horizon. In this sense, the entropy of both anisotropic and isotropic BHs are indistinguishable. Second, a critical radius appears at

$r_{c}=\frac{L^{2 / 3} \sqrt[3]{M}}{\sqrt[3]{2}}$

where the solution diverges. Even more, an analysis of the curvature scalar, which is given by

$$
\begin{aligned}
R= & -12 \alpha c_{1}-\frac{6 r}{L^{2} M-2 r^{3}}+\frac{12}{L^{2}}-\frac{8}{r^{2}} \\
& +2 \frac{2^{2 / 3}\left(2 \sqrt{3} \tan ^{-1}(A)+\log (B)\right)}{L^{4 / 3} M^{2 / 3}},
\end{aligned}
$$

reveals that this critical radius is a real singularity. It is worth noticing that, the location of $r_{c}$ is independent of the free parameters of the theory unlike the obtained in Ref. [24] and, in this sense, we can not control its location. However, the critical radius is less than the horizon radius which implies that the singularity is hidden inside the $\mathrm{BH}$ horizon. Third, the energy density reach a maximum at $r_{M}=\sqrt[3]{3 \sqrt{\frac{5}{2}} L^{2} M+5 L^{2} M}$ and its asymptotic behaviour is given by

$$
\begin{aligned}
& \lim _{r \rightarrow r_{c}} \rho \rightarrow-\infty \\
& \lim _{r \rightarrow \infty} \rho=\frac{1}{16}\left(\frac{6 c_{1} \alpha}{\pi}-\frac{2^{2 / 3} \sqrt{3}}{L^{4 / 3} M^{2 / 3}}-\frac{6}{\pi L^{2}}\right)
\end{aligned}
$$

and at the horizon

$\rho\left(r_{H}\right)=\frac{-9.49286 L^{2 / 3}+6 c_{1} \alpha L^{2} M^{2 / 3}-6 M^{2 / 3}}{16 \pi L^{2} M^{2 / 3}}$.

At this point a couple of comments are in order. First, note that near the critical radius, the apparition of exotic matter is unavoidable. Second, as far as $r \rightarrow \infty$ the choice of the free parameters leads to a negative, positive or even vanishing energy density. Even more, given that $\rho$ is a continuous function that reaches a maximum at $r_{M}$, the energy density profile could be endowed with one, two or with no real roots at all. It is worth noticing that when no real roots appear, the exotic behaviour is present in all the space-time. When two real roots are allowed, two exotic sectors appear, one near the critical point and the other for some $r>r_{c}$. Finally, for only one real root, the apparition of the exotic matter can be minimized. Specifically, one condition for the apparition of only one real root leads to

$c_{1} \alpha=\frac{6 M^{2 / 3}+9.49286 L^{2 / 3}}{6 L^{2} M^{2 / 3}}$,

which implies that the root is located at the horizon, i.e.,

$\rho\left(r_{H}\right)=0$

For this value of $c_{1} \alpha$, the energy density reach a positive value at infinity given by

$\rho \approx \frac{0.0170132}{L^{4 / 3} M^{2 / 3}}$

In Fig. 1 we show the behaviour of the energy density (blue line) and the pressure (orange line) for $r>r_{H}, c_{1} \alpha=$ $\frac{6 M^{2 / 3}+9.49286 L^{2 / 3}}{6 L^{2} M^{2 / 3}}$ and $M=L=1$, revealing that, for $r>$ $r_{H}$, all the energy conditions are satisfied.

The results obtained so far could be interpreted as follows. The isotropic sector of the polytropic $\mathrm{BH}$ reported in Ref. [34] corresponds to a $\mathrm{BH}$ solution hiding exotic matter in its interior. What is more, the new isotropic BH solution obtained in this work could be thought as the remnant of a 


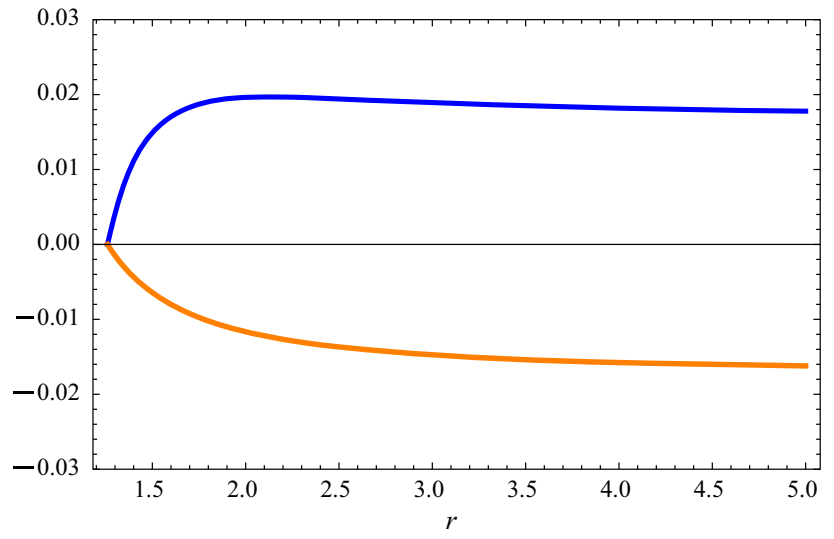

Fig. 1 Energy density (blue line) and pressure (orange line) profile for $L=1$

gravitational collapse involving exotic matter which is the responsible of sustain traversable wormholes [36-39].

\section{Conclusions}

In this work we have extended the Minimal Geometric Deformation approach when a cosmological constant is present showing that, in this case, only the isotropic sector is modified. In particular, the inverse problem in the context of polytropic black holes has been explored, obtaining the isotropic sector from which an anisotropic (A-)dS polytropic black hole is obtained. Moreover, the isotropic sector contains a singularity which does not depends on any of the free parameters of the deformation approach. This singularity is hidden inside an event horizon, which remarkably coincides with that of the anisotropic black hole. Finally, we have noted that the isotropic sector is deeply linked with the appearance of exotic matter, although it can be located inside the horizon. In this sense, this work shows a nice example of how one could, in principle, control the energy conditions by tuning the isotropy/anisotropy of a black hole solution.

Open Access This article is distributed under the terms of the Creative Commons Attribution 4.0 International License (http://creativecomm ons.org/licenses/by/4.0/), which permits unrestricted use, distribution, and reproduction in any medium, provided you give appropriate credit to the original author(s) and the source, provide a link to the Creative Commons license, and indicate if changes were made.

Funded by SCOAP ${ }^{3}$.

\section{References}

1. L. Randal, R. Sundrum, Phys. Rev. Lett. 83, 3370 (1999)

2. L. Randal, R. Sundrum, Phys. Rev. Lett. 83, 4690 (1999)

3. I. Antoniadis, Phys. Lett. B 246, 377 (1990)

4. I. Antoniadis, N. Arkani-Hamed, S. Dimopoulos, G. Dvali, Phys. Lett. B 436, 257 (1998)

5. J. Ovalle, Mod. Phys. Lett. A 23, 3247 (2008)

6. J. Ovalle, Int. J. Mod. Phys. D 18, 837 (2009)

7. J. Ovalle, Mod. Phys. Lett. A 25, 3323 (2010)

8. R. Casadio, J. Ovalle, Phys. Lett. B 715, 251 (2012)

9. J. Ovalle, F. Linares, Phys. Rev. D 88, 104026 (2013)

10. J. Ovalle, F. Linares, A. Pasqua, A. Sotomayor, Class. Quantum Gravity 30, 175019 (2013)

11. R. Casadio, J. Ovalle, R. da Rocha, Class. Quantum Gravity 31, 045015 (2014)

12. R. Casadio, J. Ovalle, Class. Quantum Gravity 32, 215020 (2015)

13. J. Ovalle, L.A. Gergely, R. Casadio, Class. Quantum Gravity 32, 045015 (2015)

14. R. Casadio, J. Ovalle, R. da Rocha, EPL 110, 40003 (2015)

15. J. Ovalle, Int. J. Mod. Phys. Conf. Ser. 41, 1660132 (2016)

16. R.T. Cavalcanti, A. Goncalves da Silva, R. da Rocha, Class. Quantum Gravity 33, 215007 (2016)

17. R. Casadio, R. da Rocha, Phys. Lett. B 763, 434 (2016)

18. J. Ovalle, Phys. Rev. D 95, 104019 (2017)

19. R. da Rocha, Phys. Rev. D 95, 124017 (2017)

20. R. da Rocha, Eur. Phys. J. C 77, 355 (2017)

21. R. Casadio, P. Nicolini, R. da Rocha, Class. Quantum Gravity 35, 185001 (2018)

22. J. Ovalle, R. Casadio, R. da Rocha, A. Sotomayor, Eur. Phys. J. C 78, $122(2018)$

23. M. Estrada, F. Tello-Ortiz, arXiv:1803.02344v3 [gr-qc]

24. J. Ovalle, R. Casadio, R. da Rocha, A. Sotomayor, Z. Stuchlik, arXiv: 1804.03468 [gr-qc]

25. C. Las Heras, P. Leon, Fortschr. Phys. 66, 1800036 (2018)

26. L. Gabbanelli, A. Rincón, C. Rubio, Eur. Phys. J. C 78, 370 (2018)

27. M. Sharif, S. Sadiq, arXiv:1804.09616v1 [gr-qc]

28. A. Fernandes-Silva, A.J. Ferreira-Martins, R. da Rocha, Eur. Phys. J. C 78, 631 (2018)

29. A. Fernandes-Silva, R. da Rocha, Eur. Phys. J. C 78, 271 (2018)

30. E. Contreras, P. Bargueño, Eur. Phys. J. C 78, 558 (2018)

31. E. Morales, F. Tello-Ortiz, arXiv: 1808.01699

32. E. Contreras, Eur. Phys. J. C 78, 678 (2018)

33. M. Estrada, R. Prado, arXiv:1809.03591

34. M. Setare, H. Adami, Phys. Rev. D 91, 084014 (2015)

35. E. Contreras, Á. Rincón, B. Koch, P. Bargueño, Eur. Phys. J. C 78, $246(2018)$

36. M.S. Morris, K.S. Thorne, Am. J. Phys. 56, 395 (1988)

37. M.S. Morris, K.S. Thorne, U. Yurtsever, Phys. Rev. Lett. 61, 1446 (1988)

38. M. Visser, Lorentzian wormholes: from Einstein to Hawking (AIP Press, New York, 1996)

39. F.S.N. Lobo, Wormholes, warp drives and energy conditions (Springer, Berlin, 2017) 